SHORT REPORT

\title{
Unreliability of triglyceride measurement to predict turbidity induced interference
}

\author{
P J Twomey, A C Don-Wauchope, D McCullough
}

J Clin Pathol 2003;56:861-862

Lipaemic specimens are a common problem in clinical chemistry. Most laboratories will measure the concentration of triglycerides and then decide whether the analytical result is valid or not. There is a poor association between the concentration of triglycerides and an objective assessment of turbidity for visually turbid specimens. Extrapolation of triglyceride concentrations derived from the use of intravenous emulsions to visually turbid specimens found in clinical practice will overestimate the turbidity induced interference in assays (non-turbid interferences are probably the same). The evaluation of turbidity induced interference needs to be standardised using objective assessments of turbidity.

ipaemic specimens are a common problem in clinica chemistry and may produce interference by three -mechanisms-light scattering, increasing the non-aqueous phase, and partitioning between the polar and nonpolar phases. ${ }^{1}$ When a lipaemic specimen is encountered, most laboratories will measure the concentration of triglycerides before deciding whether the analytical result is valid or not. Such a decision is often based on information provided in the assay data sheets provided by manufacturers. However, data may be derived from research based on either the use of an intravenous lipid emulsion (Aeroset ${ }^{\mathrm{TM}}$ System (Abbott Diagnostics). Reagent application sheet for gamma-glutamyl transferase, page 3: interfering substances. 30-1956/Rl/ February 2002) or the use of endogenous lipoproteins (Aeroset $^{\mathrm{TM}}$ System (Abbott Diagnostics). Reagent application sheet for magnesium, page 3: interfering substances. 302915/R7/August 2002). For both methods, the interference is usually reported at a given concentration of triglycerides (Aeroset $^{\mathrm{TM}}$ System. 30-1956/R1/February 2002 and Aeroset ${ }^{\mathrm{TM}}$ System. 30-2915/R7/August 2002, see above).

"When a lipaemic specimen is encountered, most laboratories will measure the concentration of triglycerides before deciding whether the analytical result is valid or not $^{\prime \prime}$

Of the three potential mechanisms for interference, the partitioning effect is analyte specific and is an infrequent problem for routine clinical chemistry analysers. The increase in the non-aqueous phase will affect all methods that do not measure an activity of the analyte. Turbidity is more likely to affect photometric methods than non-photometric methods; however, the relation between triglyceride concentrations and turbidity has previously been reported as variable, ${ }^{2}$ without comment about the degree of the variability. Furthermore, there are few data on the relation between turbidity and the source of the triglycerides; that is, endogenous lipoproteins or intravenous lipid emulsions.
Several analysers use absorbance data to calculate a lipaemic index as an objective measure of turbidity, including the Aeroset $^{\mathrm{TM}}$ system (Abbott Diagnostics) (Aeroset $^{\mathrm{TM}}$ system operations manual (Abbott Diagnostics). 30-1672/R2-March 1999, pages 2-107 to 2-108). Using the lipaemic index on the Aeroset system we investigated the relation between turbidity and triglyceride concentrations for visually turbid and nonturbid serum specimens in addition to pooled serum specimens spiked with Ivelip ${ }^{\circledR}{ }^{3}$

\section{METHODS}

Using standard Abbott Aeroset reagents and specifications, we measured the triglyceride concentration and lipaemic index (saline method used) in singleton for 35 visually turbid and 20 visually non-turbid serum specimens within two hours of routine centrifugation. Using a pooled serum specimen, Ivelip $20 \%^{3}$ was added to produce $1 \%, 2 \%, 3 \%$, $4 \%$, and $5 \%$ Ivelip $20 \%$ serum solutions. These specimens were analysed in quadruplicate for triglycerides and the lipaemic index. Deming regression equations and $r^{2}$ values were derived from these data.

\section{RESULTS}

For the visually non-turbid and visually turbid specimens the lipaemic index ranges were $0.01-0.5$ and $0.36-3.79$, respectively, and the triglyceride ranges were $1.0-4.64 \mathrm{mmol} / \mathrm{litre}$ and 3.63-40.2 $\mathrm{mmol} /$ litre, respectively (table 1 ).

The Deming regression equations and $r^{2}$ values were markedly different.

Non-turbid specimens: lipaemic index $=0.0264$ [triglycerides $]+0.0074 ; r^{2}=0.7795$

Turbid specimens: lipaemic index $=0.0479$ [triglycerides ] + 0.5608; $r^{2}=0.2399$

Ivelip specimens: lipaemic index $=2.3742$ [triglycerides $]+1.7256 ; r^{2}=0.9994$

\section{DISCUSSION}

The Aeroset lipaemic index uses three wavelength combinations $(500 / 524,572 / 604$, and 524/804 nm) to make an objective assessment of lipaemia induced turbidity (Aeroset $^{\mathrm{TM}}$ system operations manual, see above). Because this is established by using another intravenous lipid emulsion (Intralipid ${ }^{\circledR}$ ), the excellent $r^{2}$ value for Ivelip specimens is expected. However, the relatively poor $r^{2}$ for the turbid specimens implies that most of the turbidity at the above wavelengths is independent of the concentration of triglycerides. Despite the poor association between the triglyceride concentration and the lipaemic index, none of the 35 visually turbid specimens had a lipaemic index (Abbott Aeroset) greater than 4.0 (table 1).

There is also a substantial difference in the slope between the Ivelip and turbid specimens, with the slope for the turbid specimens being closer to non-turbid specimens (not shown) than the Ivelip spiked specimens (fig 1). The implication of this is that there is an increase in the light scattering for each 
Table 1 Relation between specimen type, lipaemic index, and triglyceride concentration

\begin{tabular}{|c|c|c|c|c|c|}
\hline Specimen type & Number & $\begin{array}{l}\text { Minimum } \\
\text { lipaemic index }\end{array}$ & $\begin{array}{l}\text { Maximum } \\
\text { lipaemic index }\end{array}$ & $\begin{array}{l}\text { Minimum triglyceride } \\
\text { concentration } \\
\text { (mmol/l) }\end{array}$ & $\begin{array}{l}\text { Maximum triglyceride } \\
\text { concentration (mmol/l) }\end{array}$ \\
\hline Visually non-turbi & 20 & 0.01 & 0.5 & 1.0 & 4.64 \\
\hline Visually turbid & 35 & 0.36 & 3.79 & 3.63 & 40.2 \\
\hline
\end{tabular}

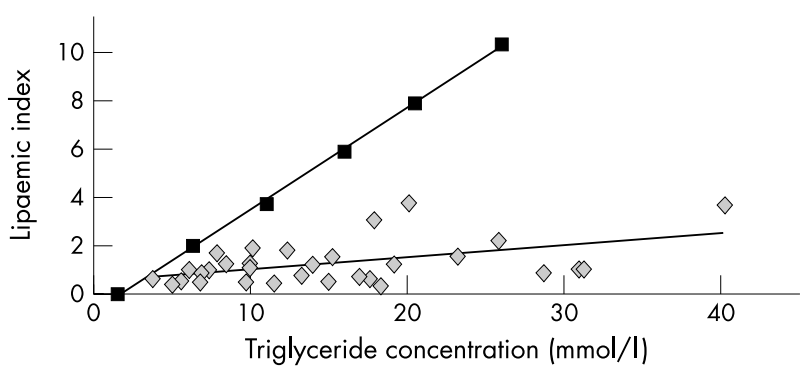

Figure 1 Triglyceride concentration versus the lipaemic index. Ivelip specimens, closed squares; turbid specimens, grey diamonds.

mmol/litre of triglycerides in the visually turbid specimens compared with visually non-turbid specimens (as expected), but that the Ivelip spiked specimens have much more light scattering for each mmol/litre of triglycerides than the visually turbid specimens. The fact that there is a difference is not surprising in view of the fact that Ivelip, like Intralipid, is a soya based lipid emulsion ${ }^{3}$ that does not contain lipoproteins. However, the degree of the difference in turbidity for each mmol/litre of triglycerides is significant if extrapolation of intravenous lipid emulsion based results is used to quantitate the degree of interference encountered in clinical practice as a result of endogenous lipoprotein based turbidity. Different analytical platforms use different wavelength parameters to determine their respective lipaemic indices. In addition, the methodologies for triglyceride assays differ for each analytical platform with respect to the type of blanking ${ }^{1}$ and error trapping. Accordingly, this effect may need to be verified for other analytical platforms.

\section{ACKNOWLEDGEMENTS}

Thanks to the staff of the Department of Clinical Biochemistry in St John's Hospital who analysed the specimens.

\section{Take home messages}

- There is a poor association between the concentration of triglycerides and an objective assessment of turbidity for visually turbid specimens

- Extrapolation of triglyceride concentrations derived from the use of intravenous emulsions to visually turbid specimens found in clinical practice will overestimate the turbidity induced interference in Abbott Aeroset ${ }^{T M}$ assays (non-turbid interferences are probably the same)

- The evaluation of turbidity induced interference should be standardised using objective assessments of turbidity

\section{Authors' affiliations}

P J Twomey, D McCullough, Clinical Biochemistry, St John's Hospital, Livingston EH54 6PP, UK

A C Don-Wauchope, Clinical Biochemistry, Royal Infirmary, Edinburgh EH3 9YW, UK

Correspondence to: Dr P J Twomey, Room S61 14, Level 2, Department of Clinical Biochemistry, 51 Little France Crescent, Edinburgh, EH16 4SA, UK; taptwomey@aol.com

Accepted for publication 27 May 2003

\section{REFERENCES}

1 Kroll MH, Elin RJ. Interference with clinical laboratory analyses. Clin Chem 1994:40:1996-2005

2 Hortin GL, Goolsby K. Lipemia interference with a rate-blanked creatinine method. Clin Chem 1997;43:408-10.

3 British National Formulary, No. 44. London: British Medical Association and Royal Pharmaceutical Society of Great Britain. ISBN 0-85369-510-5. 